\title{
Evaluation of Optimal Acquisition Duration or Injected Activity for Pediatric ${ }^{18}$ F-FDG PET/CT
}

\author{
Adam M. Alessio ${ }^{1,2}$, Marla Sammer ${ }^{3}$, Grace S. Phillips ${ }^{1,2}$, Vivek Manchanda ${ }^{1}$, Brandt C. Mohr $^{1}$, and Marguerite T. Parisi ${ }^{1,2}$ \\ ${ }^{1}$ Department of Radiology, University of Washington, Seattle, Washington; ${ }^{2}$ Department of Radiology, Seattle Children's Hospital, \\ Seattle, Washington; and ${ }^{3}$ T.C. Thompson Children's Hospital, Chattanooga, Tennessee
}

Pediatric ${ }^{18} \mathrm{~F}-\mathrm{FDG}$ dosing and acquisition durations are generally based on coarse extrapolation from adult guidelines. This study sought to determine whether shorter acquisition durations or a lower ${ }^{18} \mathrm{~F}-\mathrm{FDG}$ injected activity could be used for pediatric ${ }^{18} \mathrm{~F}-\mathrm{FDG} \mathrm{PET} / \mathrm{CT}$ examinations while maintaining diagnostic utility. Reduction of overall scan time potentially reduces motion artifacts, improves patient comfort, and decreases length of sedation. Alternatively, decreased ${ }^{18} \mathrm{~F}$ FDG dose minimizes radiation risk. Methods: Fourteen whole-body ${ }^{18} \mathrm{~F}-\mathrm{FDG}$ PET/CT examinations were performed on 13 patients (weight, 13-109 kg; age range, 1-23 y) with a weight-based injected activity $(5.3 \mathrm{MBq} / \mathrm{kg}[0.144 \mathrm{mCi} / \mathrm{kg}])$, fixed acquisition durations ( $3 \mathrm{~min} /$ field of view [FOV] if $<22$ $\mathrm{kg}, 5 \mathrm{~min} / \mathrm{FOV}$ if $>22 \mathrm{~kg}$ ), and list-mode acquisition. For each examination, the list-mode data were truncated to form multiple datasets with shorter acquisition durations down to a minimum of $1 \mathrm{~min} / \mathrm{FOV}$ (i.e., 1, 2, 3, 4, and $5 \mathrm{~min} / \mathrm{FOV}$ data were formed from single $5 \mathrm{~min} / \mathrm{FOV}$ acquisition). Fifty-six image volumes were generated, randomized, and reviewed in a masked manner with corresponding CT image volumes by 5 radiologists. Overall, subjective adequacy and objective lesion detection accuracy by body region were evaluated. Results: All examinations with maximum acquisition duration were graded as adequate and were used as the reference standard for detection accuracy. For patients less than 22 $\mathrm{kg}, 1$ of the 3 PET/CT examinations was graded as inadequate for clinical tasks when acquisition duration was reduced to $2 \mathrm{~min} / \mathrm{FOV}$, and all examinations were graded as inadequate when reduced to $1 \mathrm{~min} / F O V$. For patients more than $22 \mathrm{~kg}$, all 3-5 min/FOV studies were graded as adequate, and 2 of the 9 studies were graded as inadequate for $2 \mathrm{~min} / F O V$ studies. Lesion detection accuracy was perfect for acquisition times between $3 \mathrm{~min} / F O V$ and $5 \mathrm{~min} / F O V$ for all regions of the body. However, lesion detection became less accurate when imaging acquisition time was reduced more than $40 \%$. Conclusion: Evaluation of image volumes generated from simulated shorter acquisition durations suggests that imaging times for larger patients $(>22 \mathrm{~kg})$ can be reduced from $5 \mathrm{~min} / \mathrm{FOV}$ to $3 \mathrm{~min} / \mathrm{FOV}$ without a loss of diagnostic utility. Using decreased acquisition times as a surrogate for ${ }^{18} \mathrm{~F}-\mathrm{FDG}$ dose, ${ }^{18} \mathrm{~F}-\mathrm{FDG}$ dose can be reduced by approximately $40 \%$ when all patients were scanned for $5 \mathrm{~min} / \mathrm{FOV}$.

Received Dec. 23, 2010; revision accepted Apr. 20, 2011.

For correspondence or reprints contact: Marguerite T. Parisi, Department of Radiology M/R-5417, 4800 Sand Point Way NE, Seattle, WA 98105.

E-mail: meg.parisi@seattlechildrens.org

COPYRIGHT $\odot 2011$ by the Society of Nuclear Medicine, Inc.
Key Words: pediatric PET/CT; dosimetry; acquisition protocols; ${ }^{18} \mathrm{~F}-\mathrm{FDG}$ activity

J Nucl Med 2011; 52:1028-1034

DOI: 10.2967/jnumed.110.086579

$\mathbf{I}_{n}$ pediatric nuclear medicine, imaging protocols are generally extrapolated from adult imaging guidelines. The two primary parameters affecting image quality, injected radioisotope activity and scan duration, can vary widely on the basis of local experience and preference (1). The European Association of Nuclear Medicine (EANM) $(2,3)$ and North American societies (4) have published guidelines for pediatric injected activity in an effort to provide guidance on recommended optimal imaging protocols. This current work seeks to justify injected activity and scan durations for pediatric whole-body ${ }^{18} \mathrm{~F}-\mathrm{FDG}$ PET/CT.

An optimal ${ }^{18} \mathrm{~F}$-FDG PET/CT protocol would result in images with diagnostic quality at a minimum of risk. For ${ }^{18}$ F-FDG PET/CT, images must be of sufficient quality to perform a variety of tasks including the detection, staging, and therapeutic response monitoring of diverse diseases. Images that can perform all of these tasks without compromising clinically relevant information will be defined as having full diagnostic utility. On the other side of the equation, for pediatric imaging, there are risks associated with the scan duration and injected activity. Specifically, longer scan durations are associated with increased risk of motion artifacts, patient discomfort, and complications from potential sedation. Likewise, increased injected radioisotope activity is linked with an increased risk of radiation-induced cancer $(5,6)$. Both diagnostic utility and risk are difficult to quantify and therefore it is inherently challenging to optimize imaging protocols for any modality. Accepting these limitations, this study seeks to determine whether shorter acquisition durations or a lower ${ }^{18} \mathrm{~F}-\mathrm{FDG}$ injected activity can be used for pediatric ${ }^{18} \mathrm{~F}$-FDG PET examinations while maintaining diagnostic utility.

Several groups have presented recommendations on pediatric PET protocols and ${ }^{18}$ F-FDG dosing (7-10). Different dosing strategies are presented in Figure 1. Injected activity is often based on simple linear extrapolation from 
adult protocols. Jacobs et al. presented a discussion of pediatric dosing (11), which led to the current EANM pediatric dose card (3). The EANM recommendations attempt to normalize the effective dose from radiopharmaceuticals across all ages. These weight-independent effective doses to patients (effective dose remains roughly the same for all weight groups) do not ensure the image quality of procedures at different ages. The original 2007 EANM recommendations suggest a dose minimum of $70 \mathrm{MBq}$, which was later revised to $14 \mathrm{MBq}$ for fully 3-dimensional PET acquisitions (2).

Accorsi et al. proposed an ${ }^{18} \mathrm{~F}-\mathrm{FDG}$ dosing scheme to normalize the noise-equivalent counting rate density (NECRD) across patient ages (12). The NECR attempts to describe the number of good counts in the raw data and has been used as a surrogate for image quality (13). One primary limitation of the work of Accorsi et al. is that it assumes that the equal NECRD across patient ages will equate to equal diagnostic performance. Pediatric CT literature suggests that smaller patients require higher signal-to-noise-ratio images for the same diagnostic performance (14), because smaller patients have smaller features to resolve. Similar logic may hold true in PET, for which cancerous neoplasms scale with the size of the organ or patient, questioning the use of normalized NECRD (15).

In this work, we attempt to normalize the diagnostic utility of PET/CT image volumes across patient weights. For each patient, the list-mode data were truncated to form multiple datasets with shorter acquisition durations to a minimum of $1 \mathrm{~min} /$ field of view (FOV) (i.e., 1, 2, 3, 4, and $5 \mathrm{~min} / \mathrm{FOV}$ data were formed from single $5 \mathrm{~min} / \mathrm{FOV}$ acquisition). Similar evaluations have been performed to determine optimal acquisition parameters for obese adult patients $(16,17)$. Fifty-six image volumes were generated, randomized, and reviewed in a masked manner with corresponding CT image volumes by 5 radiologists. Overall adequacy and lesion detection accuracy by body region were evaluated.

\section{MATERIALS AND METHODS}

\section{Patients}

This study was approved by the Institutional Review Board of Seattle Children's Hospital and consisted of a population of 13 consecutive pediatric patients (14 examinations; mean age $\pm \mathrm{SD}$, $8.8 \pm 6.2 \mathrm{y}$; age range, $1-23 \mathrm{y})$ who received whole-body ${ }^{18} \mathrm{~F}-$ FDG PET/CT examinations for detection or staging of lymphoma $(n=5)$, sarcoma $(n=4)$, neuroblastoma $(n=2)$, hepatoblastoma $(n=1)$, and Castleman disease $(n=1)$. One patient, an 11-y-old boy with non-Hodgkin lymphoma, had 2 imaging studies performed. Patient weight averaged $52.1 \pm 28.2 \mathrm{~kg}$ and ranged from 13 to $109 \mathrm{~kg}$. The body mass index of the study patients averaged $21.4 \pm 6.8$ and ranged from 15.7 to 36.7 .

\section{PET/CT}

All patients were scanned using weight-based PET/CT protocols (10). Patients underwent identical preparation $(6 \mathrm{~h}$ of taking nothing by mouth except water hydration; and warm, quiescent uptake environment), were scanned $1 \mathrm{~h}$ after injection of ${ }^{18} \mathrm{~F}-\mathrm{FDG}$ and were imaged during quiet breathing. The PET preceded the CT portion, to reduce the potential for respiratory and other motion mismatch. All images were acquired on a 64-slice PET/ CT scanner (Discovery VCT; GE Healthcare) (18).

The injected ${ }^{18}$ F-FDG activity was linear with weight $(5.3$ $\mathrm{MBq} / \mathrm{kg}, 37 \mathrm{MBq}$ minimum and $370 \mathrm{MBq}$ maximum). Patients weighing more than $22 \mathrm{~kg}$ were scanned with 5 min per bed position, and patients less than $22 \mathrm{~kg}$ were scanned with $3 \mathrm{~min}$ per bed position. This shorter acquisition duration was chosen to minimize sedation time for these smaller patients, who all require sedation. The PET system has a $15.7-\mathrm{cm}$ axial FOV; with bed overlap, the effective FOV is $12.6 \mathrm{~cm}$, leading to 5-12 scanning bed positions depending on patient height. All PET data were collected in 3-dimensional mode with list-mode acquisition (each coincident event is stored in order of detection).

The CT technique was designed for attenuation correction and anatomic localization of the PET findings. All patients were scanned with a tube voltage of $120 \mathrm{kVp}$, pitch of $0.98: 1$, rotation speed of $0.5 \mathrm{~s}$, body bow-tie filter, and reconstructed slice thickness of $2.5 \mathrm{~mm}$. The maximum CT tube current (10-40 mAs) was adjusted on the basis of patient weight (10).

\section{PET Data Processing}

The portions of list-mode data from each PET acquisition were truncated to effectively form reduced-scan-duration acquisitions. We reduced scan durations for each bed position by integer values ranging from 1 to $4 \mathrm{~min}$. For example, for a larger patient, a $5 \mathrm{~min} /$ FOV data acquisition was truncated into 1, 2, 3, and $4 \mathrm{~min} / \mathrm{FOV}$ data, and for smaller patients, a $3 \mathrm{~min} / \mathrm{FOV}$ data acquisition was truncated into $1 \mathrm{~min} / \mathrm{FOV}$ and $2 \mathrm{~min} / \mathrm{FOV}$ data. The truncated data were taken from the first portion of the list-mode data. For example, the $2 \mathrm{~min} / \mathrm{FOV}$ data were taken from the first $2 \mathrm{~min}$ of the acquisition. In this manner, the datasets were not independent realizations of the data but represented both reduced total counts and reduced potential for patient movement during the scanning of a bed position. On the basis of the hypotheses that it would be difficult to see image quality differences in $4 \mathrm{~min} / \mathrm{FOV}$ studies, compared with $5 \mathrm{~min} / \mathrm{FOV}$ studies, and to simplify data manipulation and review, $4 \mathrm{~min} / \mathrm{FOV}$ studies were not generated for 6 of the $5 \mathrm{~min} / \mathrm{FOV}$ patients. From the 14 original datasets, 42 reducedscan-duration versions were generated, leading to a total of 56 datasets for evaluation. All datasets were reconstructed into image volumes with 3-dimensional ordered-subset expectation maximization ( 3 iterations, 28 subsets, 7-mm postsmoothing), with an axial FOV that included the base of the skull to the thighs in all patients.

\section{Image Evaluation}

All PET and CT image volumes were made anonymous and then interpreted by 2 reader groups, each consisting of 1 boardcertified radiologist and 1 nuclear medicine specialist. In each group, at least 1 of the readers was certified to read PET/CT scans. The order of the images was randomized and presented in order of increasing scan duration, such that the shorter-duration scans for any given patient were read first. This order was intended to present the lower-quality scans first, to avoid evaluation bias. The PET/CT scans were reviewed on a volumetric review workstation (VolumeViewer 2; GE Healthcare) to match the current clinical review steps for PET/CT at Seattle Children's Hospital. The readers used review tools and steps based on their preference. The 
FIGURE 1. Pediatric administered ${ }^{18} \mathrm{~F}-\mathrm{FDG}$ activity guidelines for whole-body PET/CT. North American consensus guidelines are based primarily on pediatric nuclear medicine experience (4), EANM dose card is based on efforts to normalize effective dose across weights (3), and Accorsi et al. presented doses based on normalized NECRD (12). $\max =$ maximum; $\min =$ minimum.

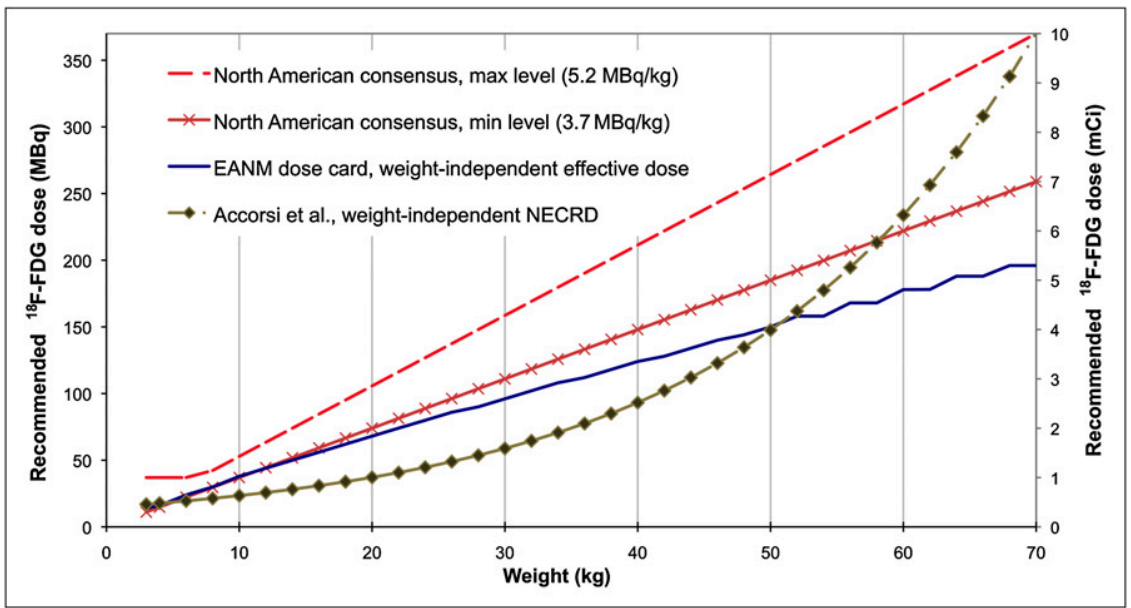

readers in groups A or B reviewed and scored each study as a team. When there was a discrepancy between groups A and B, a fifth radiologist (certified in both pediatric radiology and PET/CT review) provided a consensus read to determine the final score.

The PET/CT images were scored to determine overall adequacy and detection performance. Readers graded images (a subjective grade based on the reader's clinical experience) as having either adequate or inadequate quality for clinical tasks. Readers also recorded the number of detected lesions in each region of the body (neck, chest, abdomen, pelvis, and musculoskeleton). The detection of lesions was based on the interpretation of both the PET image and the CT image, in keeping with standard clinical PET/CT evaluation.

\section{Quality Analysis}

For the adequacy analysis, the observed agreement between reader groups $\mathrm{A}$ and $\mathrm{B}$ was evaluated for each acquisition duration. When groups A and B agreed on adequacy, their grade was the overall adequacy score; when groups A and B disagreed, the consensus reader defined the overall adequacy score.

For the detection analysis, agreement between readers was evaluated with Cohen's $\kappa$-statistic for categoric data that suggests that $\mathrm{k}$ values greater than 0.80 indicate almost-perfect concordance and $\mathrm{k}$ values of $0.61-0.80$ indicate substantial agreement (19). For these studies, the detection results from the longestduration scan were defined as truth for the patient. The accuracy of detection for each body region was reported in terms of truepositive rates and false-positive rates.

\section{RESULTS}

Examples of PET/CT image volumes generated with reduced PET acquisition duration are presented in Figures 2 and 3. As expected, there is increased noise in the shorterduration scans.

\section{Adequacy}

The adequacy scores for readers $\mathrm{A}$ and $\mathrm{B}$ and the consensus reader are summarized in Table 1. There was strong agreement between the readers for scans with acquisition times of $5 \mathrm{~min} / \mathrm{FOV}$ down to $2 \mathrm{~min} / \mathrm{FOV}$. All readers scored the longest-duration scan as adequate in all patients. On the basis of the consensus read, all 3 and $4 \mathrm{~min} / \mathrm{FOV}$ scans were also scored as adequate. Only $75 \%$ of the $2 \mathrm{~min} / \mathrm{FOV}$ scans and $30 \%$ of the $1 \mathrm{~min} / \mathrm{FOV}$ scans were considered adequate. The overall adequacy scores for each patient are presented in Figure 4 with different marker types. Four patients were scored as adequate for all scan durations, and on the other end of the spectrum, 3 patients were scored as inadequate for their 2-min acquisitions.

\section{Lesion Detection}

Forty-nine lesions were detected on the full-duration scans. Of these, 15 were in the neck, 7 in the chest, 9 in the abdomen, 9 in the pelvis, and 9 in bone. All patients, except 1 , had at least 1 lesion in 1 body region. The false-positives and false-negatives for each patient for any body region are shown in Figure 4; the labels for false-positive or falsenegative represent one or more detection failures. The true-positive and false-positive rates are plotted in Figure 5 by body region on the basis of scores from the consensus reader. Although there was some disagreement in the total number of lesions, there was no disagreement in the assessment of disease present versus disease absent in each body
$A$

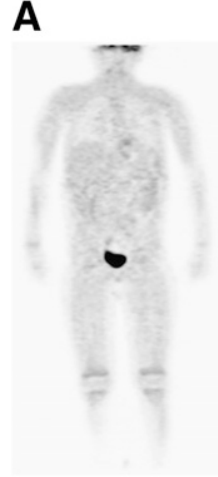

B

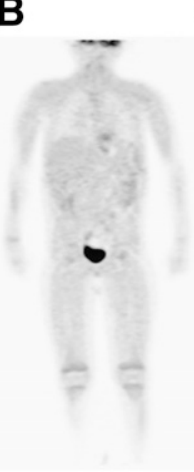

C

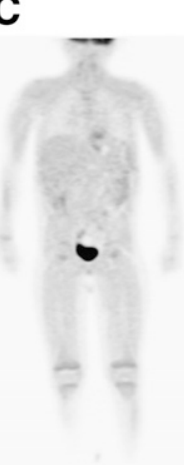

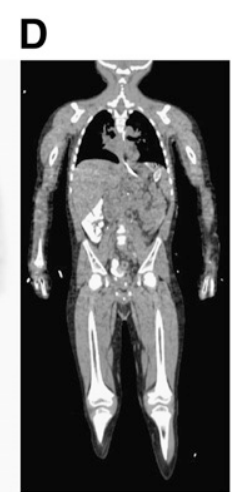

FIGURE 2. Example coronal views of ${ }^{18} \mathrm{~F}-\mathrm{FDG}$ PET/CT examination of $17-\mathrm{kg}$ patient with $1 \mathrm{~min} / \mathrm{FOV}(\mathrm{A}), 2 \mathrm{~min} / \mathrm{FOV}(\mathrm{B})$, and $3 \mathrm{~min} / \mathrm{FOV}(\mathrm{C})$ acquisition duration. Coronal view of CT image is presented in D. For this patient, $3 \mathrm{~min} / \mathrm{FOV}$ was graded as adequate, whereas $2 \mathrm{~min} / \mathrm{FOV}$ and $1 \mathrm{~min} / \mathrm{FOV}$ were graded as inaccurate. All PET images are shown with matched window levels. 


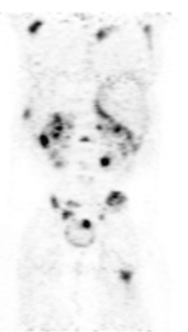

B

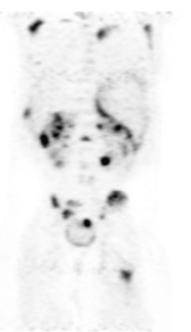

\section{C}

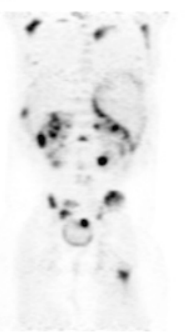

D

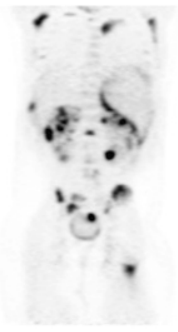

FIGURE 3. Example coronal views of FDG PET/CT examination of 61-kg patient with $1 \mathrm{~min} / \mathrm{FOV}(\mathrm{A}), 2 \mathrm{~min} / \mathrm{FOV}(\mathrm{B}), 3 \mathrm{~min} / \mathrm{FOV}(\mathrm{C})$, and $5 \mathrm{~min} / \mathrm{FOV}$ (D) acquisition duration. For this patient, all acquisition durations were graded as adequate. Images are shown with matched window levels.

region for the 5, 4, and $3 \mathrm{~min} / \mathrm{FOV}$ images. False-positives occurred in the neck (61-kg patient), chest region (94-kg patient), and pelvis (same 94-kg patient) for the $2 \mathrm{~min} / \mathrm{FOV}$ studies, and true-positive rate degrades for most regions for the $1 \mathrm{~min} / \mathrm{FOV}$ studies.

The agreement in number of detected lesions between readers $\mathrm{A}$ and $\mathrm{B}$ is presented in Table 2. For body regions other than the chest, there was excellent agreement between readers for the $5,4,3$, and $2 \mathrm{~min} / \mathrm{FOV}$ images, as demonstrated by $\kappa$-scores greater than 0.7 . The readers had moderate agreement in the chest region, for which there was larger discrepancy between readers because of different interpretations of mediastinal ${ }^{18} \mathrm{~F}-\mathrm{FDG}$ activity, where 1 group reported all suggestive foci and the other group discounted foci with no pathologic significance (i.e., esophagitis in a postchemotherapeutic patient or mild, diffuse ${ }^{18} \mathrm{~F}-\mathrm{FDG}$ activity in the hilum). In general, there was demonstrably less agreement in the $1 \mathrm{~min} / \mathrm{FOV}$ images. Readers A and B defined disease present (1 lesion or more) and disease absent (no lesions) identically in all regions and all studies for scans of $5 \mathrm{~min} / \mathrm{FOV}$ down to $3 \mathrm{~min} / \mathrm{FOV}$, except in 1 case in which reader $\mathrm{B}$ had a false-positive result in the abdomen for a 3-min scan. Consequently, for 5- down to 3-min studies, the deviation from perfect agreement presented in Table 2 signifies slight variations in the number of detected lesions in each body region. The $\kappa$-score could not be computed in the cases with zero lesions (abdomen, 4-min duration).

\section{DISCUSSION}

This study evaluates subjective image quality and objective detection performance for reduced-scan-duration pediatric whole-body ${ }^{18} \mathrm{~F}-\mathrm{FDG}$ PET/CT. For examinations in patients more than $22 \mathrm{~kg}(n=11)$, the adequacy of scans was not degraded when the scans were reduced from 5- to 3-min acquisitions. For these larger patients, the detection task performance is identical for 3- to 5-min acquisitions and is slightly degraded for 2-min acquisitions. When a threshold of less than $22 \mathrm{~kg}$ was used to define the class of patients that require sedation during scanning, for these smallest patients $(n=3)$, the adequacy and detection performance of scans could be assured only at 3-min per bed position. In particular, for the $2 \mathrm{~min} / \mathrm{FOV}$ study of the $17-\mathrm{kg}$ patient, all readers deemed the study as inadequate and there was a false-negative for lesions in the pelvic region. Because this was a consistent failure for all readers, we consider this a suboptimal scan. In this work, because of the limited numbers of subjects, we adopted the conservative approach and claimed that any degradation in overall adequacy or detection performance would dictate the accepted performance for that patient group. As a result, these results suggest that acquisitions can be reduced to $3 \mathrm{~min}$ per bed position for patients more than $22 \mathrm{~kg}$ ( $40 \%$ reduction in scan time) and that the protocol should remain the same ( $3 \mathrm{~min}$ per bed position) for patients less than $22 \mathrm{~kg}$.

Reducing acquisition duration or injected radiotracer activity can lead to identical PET images. In the absence of detector dead time, the number of true coincident events decreases linearly with decreases in injected activity. The number of scattered events decreases linearly, and the number of random events decreases with the square of decreased injected activity (20). When these are combined with detector dead time, the ratio of true events to degrading events decreases more than linearly with injected activity. For this reason, if a $30 \%$ reduction in acquisition duration provides diagnostic utility equivalent to the fullduration scan, then it suggests that a $30 \%$ reduction in injected activity (at the full-duration scan) will provide higher image quality. The exact relationship of scan duration reduction to injected activity reduction is a complex function of the size of the patient, scan region, and counting

TABLE 1

Summary of Adequacy Scores for Each Acquisition Duration

\begin{tabular}{|c|c|c|c|c|c|c|}
\hline \multirow[b]{2}{*}{ Parameter } & \multirow[b]{2}{*}{ All studies } & \multicolumn{5}{|c|}{ Acquisition duration (min/FOV) } \\
\hline & & 1 & 2 & 3 & 4 & 5 \\
\hline Number of image volumes & 56 & 14 & 12 & 14 & 5 & 11 \\
\hline Observed agreement (\%) & 88 & 64 & 92 & 93 & 100 & 100 \\
\hline Mean score of readers A and B (proportion acceptable) & 0.79 & 0.39 & 0.79 & 0.96 & 1 & 1 \\
\hline Mean score after consensus read (proportion acceptable) & 0.77 & 0.29 & 0.75 & 1 & 1 & 1 \\
\hline
\end{tabular}

Mean score was derived from average of readers $A$ and $B$ at each acquisition duration. Consensus mean score was derived from average at each acquisition duration. 
FIGURE 4. Summary of results for all acquisition durations $(n=56)$ for all patients. Each marker represents PET/CT image set. One or more lesion detection failures are noted as false-positive and false-negative below marker for that study. $\bigcirc=$ studies deemed as adequate for interpretation; $x=$ studies deemed as inadequate; $\mathrm{FN}=$ falsenegative; FP = false-positive.

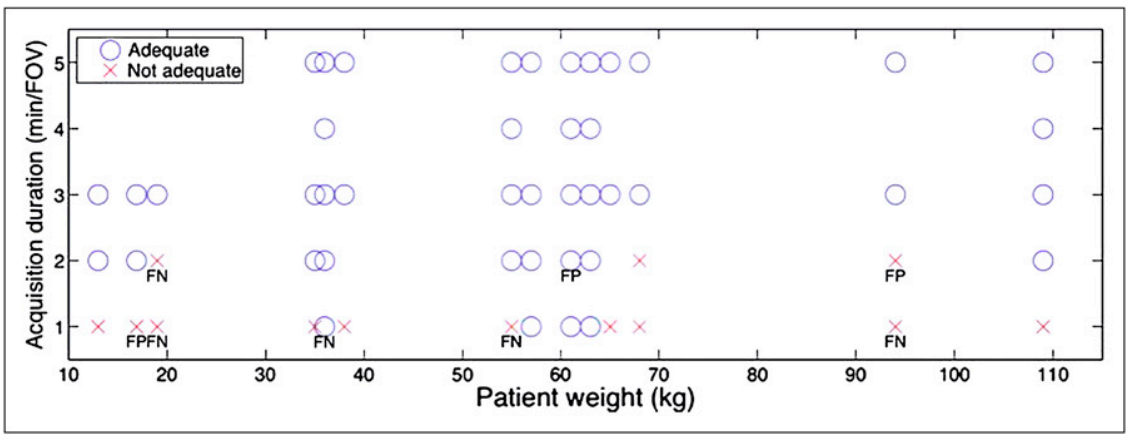

rate performance of the imaging system (21). Therefore, we suggest the conservative relationship that reductions in acquisition duration can be directly traded off for reductions in activity. Consequently, the $40 \%$ reduction in acquisition duration for patients more than $22 \mathrm{~kg}$ could be traded for a $40 \%$ reduction in injected activity. Results suggest that the new protocol for patients more than $22 \mathrm{~kg}$ could be an injected activity of $3.2 \mathrm{MBq} / \mathrm{kg}(0.09 \mathrm{mCi} / \mathrm{kg})$ with a scan duration of $5 \mathrm{~min} / \mathrm{FOV}$.

These proposed changes in injected activity that provide diagnostic utility equivalent to the full-duration scans are presented in Figure 6. The proposed dosing of $3.2 \mathrm{MBq} / \mathrm{kg}$ is close to the minimum level of $3.7 \mathrm{MBq} / \mathrm{kg}$ suggested in the North American consensus guidelines (4). These results provide image quality confirmation of the minimum linear relationship from the North American guidelines and suggest that the maximum dose could be changed from 370 $\mathrm{MBq}(10 \mathrm{mCi})$ to $222 \mathrm{MBq}(6 \mathrm{mCi})$. Likewise, these results are close to the EANM recommendations.
In general, dosing schemes were originally devised to minimize radiation dose to critical organs, with little effort to optimize image quality. At present, none of the ${ }^{18}$ F-FDG PET recommendations for adult or pediatric dosing in the literature include firm recommendations on optimal scan durations. If our current protocols $(5.3 \mathrm{MBq} / \mathrm{kg}, 5 \mathrm{~min} / \mathrm{FOV})$ are assumed to have appropriate image quality, this study suggests that a dosing scheme of $3.2 \mathrm{MBq} / \mathrm{kg}$ with $5 \mathrm{~min} /$ FOV will provide equivalent image quality. Along with limitations of the study, this claim may not hold with different PET/CT systems (each with unique sensitivity performance) and with different readers (each with unique interpretation preferences). Despite these challenges, we assert that there is value to providing guidance to pediatric scanning protocols based on measures of image quality.

Limitations of this study include the subjective evaluation of image quality, limited number of patients enrolled, and assumption of diagnostic utility of the full-duration scan. The determination of image quality is challenging
FIGURE 5. Average detection performance for different regions at different acquisition durations. FPR $=$ false-positive rate; TPR $=$ true-positive rate.

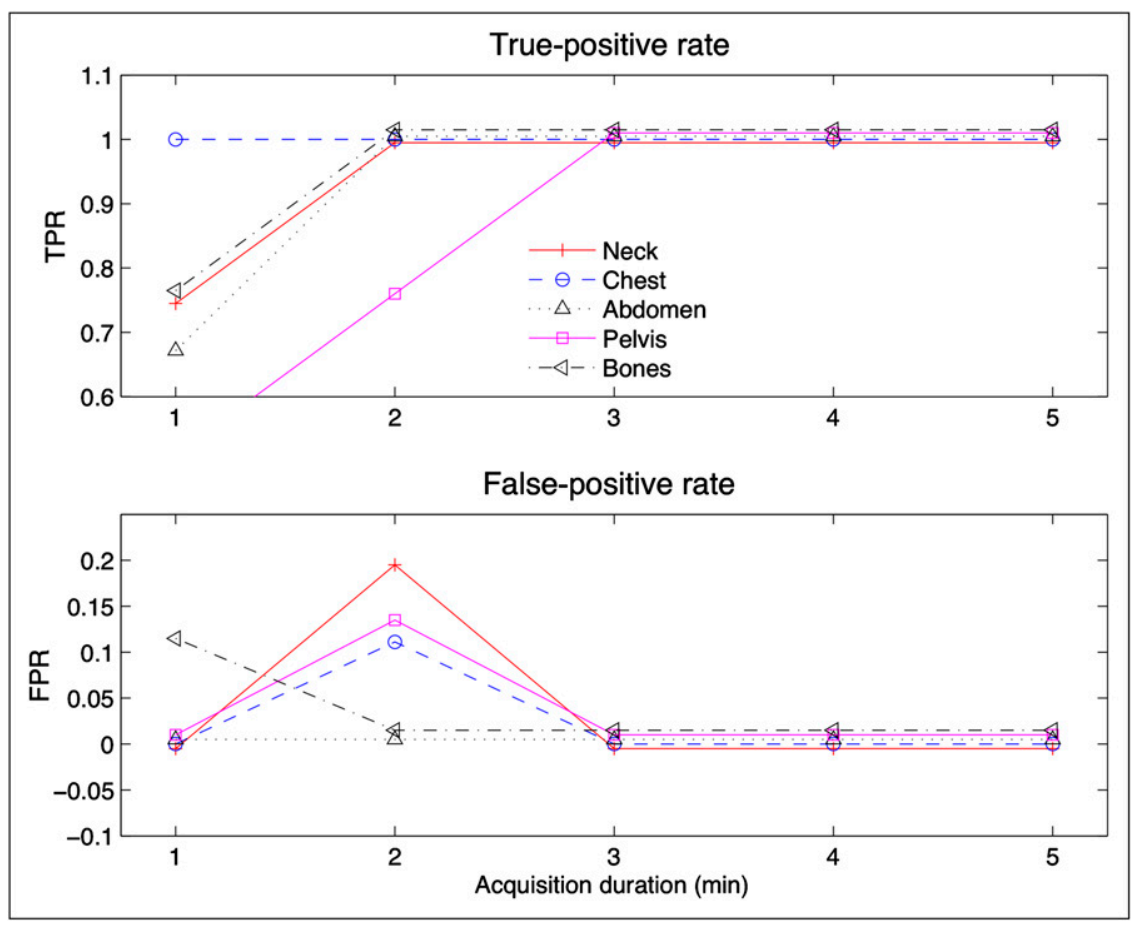


TABLE 2

Summary of Lesion Location and Cohen's к-Coefficient Between Readers A and B for Number of Detected Lesions in Each Region

\begin{tabular}{|c|c|c|c|c|c|c|c|c|}
\hline & \multirow[b]{2}{*}{ No. of patients with lesions } & \multirow[b]{2}{*}{ Total no. of lesions } & \multirow[b]{2}{*}{$\kappa$, all studies } & \multicolumn{5}{|c|}{$\kappa$ for each acquisition duration (min/FOV) } \\
\hline & & & & 1 & 2 & 3 & 4 & 5 \\
\hline Neck & 8 & 15 & 0.79 & 0.48 & 0.85 & 0.85 & 1 & 1 \\
\hline Chest & 5 & 7 & 0.51 & 0.36 & 0.54 & 0.58 & 1 & 0.47 \\
\hline Abdomen & 3 & 9 & 0.85 & 0.81 & 0.79 & 0.82 & - & 1 \\
\hline Pelvis & 4 & 9 & 0.86 & 0.73 & 0.67 & 1 & 1 & 1 \\
\hline Bones & 4 & 9 & 0.96 & 0.85 & 1 & 1 & 1 & 1 \\
\hline
\end{tabular}

(22). In this work, we sought to determine the diagnostic equivalence of reduced-scan- and full-scan-duration studies. In general, quantitative human or numeric observer studies based on simulation or numerous patient scans would provide more precise results than our current study, but they would require a more restrictive experimental design (such as the presentation of single PET slices or PET-only image volumes) that does not mimic clinical practice. We chose to re-create the clinical task of a dualmodality PET/CT review in which the PET and CT provide complementary information for interpretation. In this realistic situation, we can claim only subjective image quality and detection equivalence. We were unable to conclude a quantitative number on the exact reduction in image quality for reductions in scan duration. Similarly, subjective image quality assessment depends on the preference of the reader and can vary widely from reader to reader. We chose the use of 5 readers to minimize this variation. Furthermore, we should stress that this study evaluated diagnostic utility in the context of PET/CT review, for which the CT scan can provide valuable side information; these results are arguably not applicable to PET-only systems.

Generally, image quality evaluations require more patients to determine exact changes in quality. We did not report numeric changes in quality, only diagnostic equivalence of studies. Diagnostic equivalence was further sup-

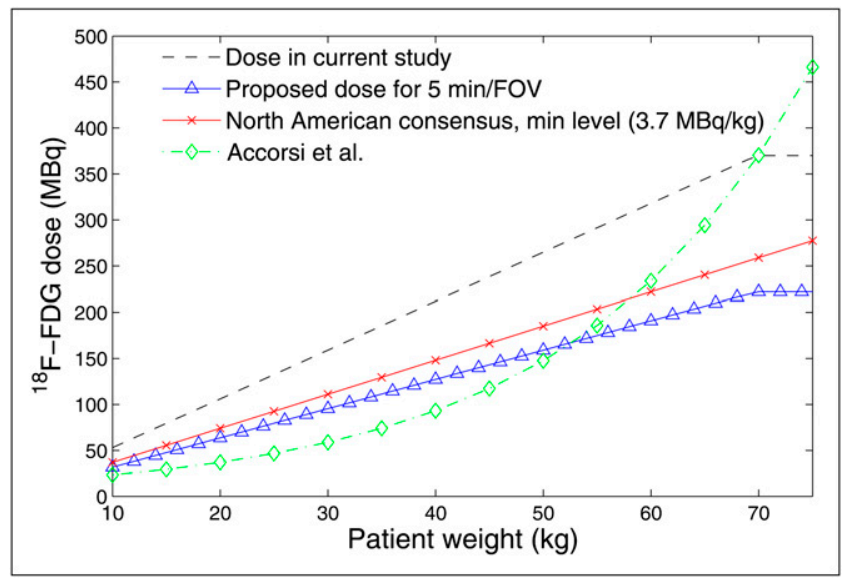

ported by the strong agreement between readers for studies with diagnostic performance equivalent to the full-duration scan. This study used coarse 1-min changes in acquisition duration to find the threshold of acceptability. More patients, with finer variations in acquisition duration, might allow us to further shorten acquisition duration or reduce injected activity.

The highest-quality PET images are generally achieved by scanning every patient for as long as is practical, regardless of dose. The only caveats to this rule are when scan time is limited by radiopharmaceutical redistribution or decay or when patient motion is excessive. Despite the inherent value of scanning for as long as possible, this approach is not always in the patient's best interest, particularly for sedated pediatric patients. Moreover, the realities of resource use limit the duration of many PET/CT acquisitions. Consequently, centers operate with image quality that is less than ideal. Our current work assumes that the full-duration scan provided full diagnostic utility - a solid working assumption given that our full acquisition duration is arguably longer than that used in many PET centers. Nevertheless, this assumption is a limitation of our current study, and our preference for full diagnostic utility may be different from other centers. The proposed dosing scheme should be confirmed by imaging centers to ensure acceptable image quality.

FIGURE 6. Administered ${ }^{18}$ F-FDG activity for different patient weights. Dose in current study used linear $5.3 \mathrm{MBq} / \mathrm{kg}$ dosing scheme with shorter acquisition durations for smaller patients. This study suggests that acquisition duration or dose can be reduced for patients more than $22 \mathrm{~kg}$. These reductions result in doses similar to minimum recommended levels of North American consensus. 
Another limitation is that image reconstruction parameters were fixed for all studies and were not optimized for the different acquisition durations. It is conceivable that different implicit (number of iterations and subsets) and explicit (postreconstruction filtering) regularization schemes would lead to better performance. In particular, more smoothing in the shorter-acquisition-duration scans would most likely improve detection performance. Consequently, optimizing reconstruction parameters might allow further reductions in acquisition duration or injected activity.

\section{CONCLUSION}

The evaluation of image volumes generated from shorter acquisition durations suggests that injected activity of 3.2 $\mathrm{MBq} / \mathrm{kg}(0.09 \mathrm{mCi} / \mathrm{kg})$ with imaging durations of $5 \mathrm{~min} /$ FOV in all patients provides PET/CT studies with diagnostic utility equivalent to that of studies with $5.3 \mathrm{MBq} / \mathrm{kg}$ $(0.14 \mathrm{mCi} / \mathrm{kg})$ for $5 \mathrm{~min} / \mathrm{FOV}$ for patients more than $22 \mathrm{~kg}$ and $3 \mathrm{~min} / \mathrm{FOV}$ for patients less than $22 \mathrm{~kg}$. In support of this conclusion, we found no reduction in subjective score or detection performance for patients more than $22 \mathrm{~kg}$ when acquisition durations were reduced from $5 \mathrm{~min} / \mathrm{FOV}$ to $3 \mathrm{~min} / \mathrm{FOV}$. For the 3 patients less than $22 \mathrm{~kg}, 1$ study had diminished subjective and detection performance at a $33 \%$ reduction in scan time. On the basis of these results and clinical experience, we suggest a conservative approach to image smaller patients with adequate activity or scan duration $(3.2 \mathrm{MB} / \mathrm{kg}$ at $5 \mathrm{~min} / \mathrm{FOV}$ or $5.3 \mathrm{MBq} / \mathrm{kg}$ at $3 \mathrm{~min} / \mathrm{FOV}$ ). Future work with additional patients may help clarify the dosing regime in this smaller population. Our results are close to the minimum recommended levels of both the North American and EANM pediatric guidelines and provide image quality confirmation of these recommendations.

\section{DISCLOSURE STATEMENT}

The costs of publication of this article were defrayed in part by the payment of page charges. Therefore, and solely to indicate this fact, this article is hereby marked "advertisement" in accordance with 18 USC section 1734.

\section{ACKNOWLEDGMENTS}

We thank Lisa Aldape and the nuclear medicine technologists at Seattle Children's Hospital for their help in acquiring and processing the PET/CT data. This study was partially supported by a grant from the Fighting Children's Cancer
Foundation. No other potential conflict of interest relevant to this article was reported.

\section{REFERENCES}

1. Treves ST, Davis RT, Fahey FH. Administered radiopharmaceutical doses in children: a survey of 13 pediatric hospitals in North America. J Nucl Med. 2008;49:1024-1027.

2. Lassmann M, Biassoni L, Monsieurs M, Franzius C. The new EANM paediatric dosage card: additional notes with respect to F-18. Eur J Nucl Med Mol Imaging. 2008;35:1666-1668.

3. Lassmann M, Biassoni L, Monsieurs M, Franzius C, Jacobs F. The new EANM paediatric dosage card. Eur J Nucl Med Mol Imaging. 2007;34:796-798.

4. Gelfand MJ, Parisi MT, Treves ST. Pediatric radiopharmaceutical administered doses: 2010 North American consensus guidelines. J Nucl Med. 2011;52: 318-322.

5. Health Risks from Exposure to Low Levels of Ionizing Radiation: BEIR VII Phase 2. Washington, DC: National Academies Press; 2006. Available at: http://www.nap. edu/catalog.php?record_id=11340. Accessed June 13, 2011.

6. Fahey FH. Dosimetry of pediatric PET/CT. J Nucl Med. 2009;50:1483-1491.

7. Barrington SF, Begent J, Lynch T, et al. Guidelines for the use of PET-CT in children. Nucl Med Commun. 2008;29:418-424.

8. Kaste SC. Issues specific to implementing PET-CT for pediatric oncology: what we have learned along the way. Pediatr Radiol. 2004;34:205-213.

9. Stauss J, Franzius C, Pfluger T, et al. Guidelines for ${ }^{18} \mathrm{~F}-\mathrm{FDG}$ PET and PET-CT imaging in paediatric oncology. Eur J Nucl Med Mol Imaging. 2008;35:1581-1589.

10. Alessio AM, Kinahan PE, Manchanda V, Ghioni V, Aldape L, Parisi MT. Weight-based, low-dose pediatric whole-body PET/CT protocols. J Nucl Med. 2009;50:1570-1577.

11. Jacobs F, Thierens H, Piepsz A, et al. Optimised tracer-dependent dosage cards to obtain weight-independent effective doses. Eur J Nucl Med Mol Imaging. 2005;32:581-588.

12. Accorsi R, Karp JS, Surti S. Improved dose regimen in pediatric PET. J Nucl Med. 2010;51:293-300.

13. Watson CC, Casey ME, Bendriem B, et al. Optimizing injected dose in clinical PET by accurately modeling the counting-rate response functions specific to individual patient scans. $J$ Nucl Med. 2005;46:1825-1834.

14. Boone JM, Geraghty EM, Seibert JA, Wootton-Gorges SL. Dose reduction in pediatric CT: a rational approach. Radiology. 2003;228:352-360.

15. Watson CC. Injected dose in pediatric PET. J Nucl Med. 2010;51:1657.

16. Halpern BS, Dahlbom M, Auerbach MA, et al. Optimizing imaging protocols for overweight and obese patients: a lutetium orthosilicate PET/CT study. J Nucl Med. 2005;46:603-607.

17. Halpern BS, Dahlbom M, Quon A, et al. Impact of patient weight and emission scan duration on PET/CT image quality and lesion detectability. J Nucl Med. 2004; 45:797-801.

18. Teras M, Tolvanen T, Johansson JJ, Williams JJ, Knuuti J. Performance of the new generation of whole-body PET/CT scanners: Discovery STE and Discovery VCT. Eur J Nucl Med Mol Imaging. 2007;34:1683-1692.

19. Landis JR, Koch GG. The measurement of observer agreement for categorical data. Biometrics. 1977;33:159-174.

20. Strother SC, Casey ME, Hoffman EJ. Measuring PET scanner sensitivity: relating countrates to image signal-to-noise ratios using noise equivalents counts. IEEE Trans Nucl Sci. 1990;37:783-788.

21. Lartizien C, Comtat C, Kinahan PE, Ferreira N, Bendriem B, Trebossen R. Optimization of injected dose based on noise equivalent count rates for 2- and 3-dimensional whole-body PET. J Nucl Med. 2002;43:1268-1278.

22. Badawi RD, Dahlbom M. NEC: some coincidences are more equivalent than others. J Nucl Med. 2005;46:1767-1768. 